\section{EU-Zulassung für Vectibix zur Behandlung des metastasierten kolorektalen Karzinoms}

Die europäische Arzneimittelagentur EMEA hat am 3. Dezember 2007 die vorläufige Zulassung für Vectibix ${ }^{\circledR}$ (Panitumumab) erteilt. Der voll humane Anti-EGFr-Antikörper ist als Monotherapie indiziert nach Versagen einer Standard-Chemotherapie bei metastasiertem kolorektalen Karzinom, das den epidermalen Wachstumsfaktor (EGFr) exprimiert und ein nicht mutiertes KRASGen (Wildtyp) aufweist.

Die Entscheidung der EU-Kommission basiert auf einem positiven Votum des Ausschusses für Humanarzneimittel CHMP vom September 2007. Der Ausschuss kam dabei zu einer positiven Nutzen/Risiko-Einschätzung von Vectibix ${ }^{\circledR}$ bei einer Patientengruppe, der derzeit nur wenige Behand- lungsoptionen verbleiben. Im Rahmen der CHMP-Prüfung wurden klinische Daten zur Verfügung gestellt, die die Verwendung des KRASMutationsstatus als Biomarker für die zu erwartenden klinischen Ergebnisse unterstützen.

Die Daten zum Biomarker KRAS wurden in einer prospektiven Auswertung der randomisierten, kontrollierten Phase-III-Studie 408 gewonnen, die den Einfluss des KRAS-Status (mutiert vs. nicht mutiert) bei Vectibix ${ }^{\circledR}$-Patienten mit metastasiertem kolorektalem Karzinom (mCRC) untersuchte. Die Analyse machte deutlich, dass der Einfluss von Panitumumab auf das progressionsfreie Überleben ausschließlich auf die Patienten (ca. 60\%) beschränkt war, deren Tumoren ein nicht-mutiertes KRAS-Gen (Wildtyp) aufwiesen. Tumoren mit mutiertem KRAS-Gen sprechen nicht auf eine Anti-EGFr-Therapie wie Panitumumab an, unabhängig vom Endpunkt der Studie. Weitere zentra- le, zuvor ausgewertete Daten der Studie 408 zeigen, dass eine Monotherapie mit Vectibix ${ }^{\circledR}$ die progressionsfreie Überlebenszeit und die Ansprechraten bei stark vorbehandelten Patienten mit einem mCRC nach dem Versagen der Standard-Chemotherapie im Vergleich zur besten supportiven Behandlung signifikant verbessert.

Weitere Informationen bei AMGEN GmbH

Silvia Schmidt

Manager Kommunikation

Tel. +49 891490 96-1602, Fax -2602

schmidts@amgen.com

\section{Taxotere erhält Zulassungserweite- rung der EMEA: Induktionstherapie bei operablen Kopf-Hals Tumoren}

Die Induktionschemotherapie mit Docetaxel (Taxotere $\left.{ }^{\circledR}\right)$, Cisplatin und 5-FU (TPF-Regime) ist eine wirksame und gut verträgliche Therapie für Patienten mit lokal fortgeschrittenem Plattenepithelkarzinom des Kopf-Hals-Bereichs (SCCHN). Bereits 2006 wurde das TPF-Regime für die Behandlung inoperabler Patienten mit T3/T4-Tumoren zugelassen. Sowohl die europäische Zulassungsbehörde EMEA als auch die US-amerikanische Zulassungsbehörde FDA haben die Zulassung jetzt auf die Induktionstherapie operabler Patienten erweitert

Die Zulassung für Docetaxel als Induktionstherapie bei operablen Patienten basiert auf den Ergebnisse der TAX 324-Studie, einer international durchgeführten randomisierten Phase-III-
Studie bei 538 Patienten mit lokal fortgeschrittenem SCCHN der Stadien T3/4 (Posner et al. ASCO 2006; Hitt et al., ASCO 2006, \#5515) Die Induktionsbehandlung mit dem TPF-Regime erreichte in dieser Studie einen statistisch signifikanten Überlebensvorteil im Vergleich zur alten Induktionstherapie mit Cisplatin/5-FU (PFRegime). Die mit TPF behandelten Patienten überlebten im Median mehr als doppelt so lange wie jene im PF-Arm (70,6 vs. 30,1 Monate). Das bedeutet eine Reduktion des relativen Sterberisikos um 30\% (HR 0,70; p = 0,0058). Nach dre Jahren waren noch zwei Drittel der TPF-Patienten am Leben (62 vs. 48\%). Signifikante Vorteile für das TPF-Regime zeigten sich auch bei der progressionsfreien Überlebenszeit mit einer relativen Risikoreduktion von $29 \%$ (HR 0,71; p $=0,004)$.

Die Ergebnisse der TAX 324-Studie werden durch die Daten der TAX 323-Studie untermau- ert, einer randomisierten Phase III-Studie, die 2006 zur Zulassung des TPF-Regimes bei inoperablen Patienten mit lokal fortgeschrittenem SCCHN geführt hatte (Remenar et al., ASCO 2006, \#5516). Hier erreichte das TPF-Regime bei primär inoperablen Patienten einen statistisch signifikanten Überlebensvorteil im Vergleich zum PF-Regime (18,6 vs. 14,2 Monate; HR 0,73; $\mathrm{p}=0,0053)$. Die progressionsfreie Zeit wurde um drei Monate verlängert (11,2 vs. 8,2 Monate; HR 0,74; $\mathrm{p}=0,015)$. Beide Studien zeigen somit, dass die TPF-Induktionschemotherapie der bisherigen Induktionschemotherapie mit PF überlegen ist.

Cristina Iannazzo

Sanofi-Aventis Deutschland $\mathrm{GmbH}$

Brand \& Scientific Communication

65926 Frankfurt am Main

Tel. +49 69 305-244 17, Fax -188 02

presse@sanofi-aventis.com

\title{
PharmaTicker+++ PharmaTicker+++ PharmaTicker+++ PharmaTicker+++
}

Roche Pharma AG. Der Ausschuss für Humanarzneimittel der Europäischen Arzneimittelagentur hat im November 2007 die Zulassungsempfehlung für Avastin ${ }^{\circledR}$ (Bevacizumab) zur Firstline-Therapie des metastasierten Nierenzellkarzinoms ausgesprochen. Die Empfehlung basiert auf den Ergebnissen der AVOREN-Studie, die zeigen, dass Bevacizumab das progressionsfreie Überleben von Patienten mit mRCC im Median auf über 10 Monate verdoppelt.

Roche Pharma AG

Dr. H.-U. Jelitto

Tel. +49 7624 14-3715, Fax -3366

www.roche.de
Pierre Fabre Pharma GmbH. Die Ergebnisse der internationalen GLOB3-Studie (Global Lung Oncology Branch trial 3) zeigen, dass die kombinierte intravenöse/orale Chemotherapie mit Vinorelbin (Navelbine ${ }^{\circledR}$ ) in der Kombination mit Cisplatin gleichwertig hinsichtlich Wirksamkeit und Verträglichkeit gegenüber der Therapie mit Docetaxel und Cisplatin ist.

Weitere Informationen bei

Publicis Vital PR

Oliver Ehrnstorfer

Tel. +49 221 912719-18, Fax -7

oliver.ehrnstorfer@publicis-vital-pr.de
Bayer Schering Pharma AG. Zevalin verlängert als Konsolidierungstherapie beim fortgeschrittenen follikulären Lymphom die Zeit bis zum Fortschreiten der Krankheit signifikant. Diese zeigten Ergebnisse der FIT-Studie (FirstLine Indolent Trial), die bei der 49. Jahresversammlung der American Society of Hematology erstmals einem wissenschaftlichen Publikum vorgestellt wurden.

Weitere Informationen bei

Bayer Schering Pharma AG

Dr. Jost Reinhard

Tel. +49 3046815062

jost.reinhard@bayerhealthcare.com 
Trends in Medical Mycology 2007

\section{Invasive Candida-Infektionen: Anidulafungin wirkt signifikant besser als Fluconazol}

Anidulafungin (Ecalta ${ }^{\circledR}$ ) ist bei Candidämie und anderen invasiven Candida-Infektionen signifikant besser wirksam als Fluconazol. Dieses Ergebnis einer im Juni im New England Journal of Medicine publizierten Studie [1] stand im Mittelpunkt des Vortrags von Prof. Coleman Rotstein [2], McMaster University, Hamilton, Ontario/Kanada, anlässlich des integrierten Symposiums «Managing Candidemia - What's New in 2007?» auf dem 3. TIMM-Kongress (Trends in Medical Mycology) Ende Oktober 2007 in Turin.

Die Behandlung mit Anidulafungin wirkte in der Primärauswertung der Studie bei Ende der intravenösen Therapie und in der Nachbeobachtung 2 Wochen nach Ende der Therapie signifikant besser als Fluconazol, der bisherige Standard in dieser Indikation (klinische und mikrobiologische Erfolgsrate 75,6 vs. $60,2 \%$ ). Die Analyse wichtiger Subpopulationen wie Intensivpatienten sowie Patienten mit Beatmung, Leberinsuffizienz oder Dialysebehandlung, ergab ebenfalls deutlich höhere Erfolgsraten bei der Therapie mit Anidulafungin (69 vs. $46 \%$; 79 vs. $57 \%$; 73 vs. $53 \%$ und 71 vs. $39 \%$ ). Dabei wurde der Erreger unter Anidulafungin tendenziell schneller aus dem Blutstrom eradiziert als dies unter Fluconazol der Fall war. Wie Rotstein betonte, sei erstmals in einer randomisierten Studie zur Therapie invasiver Candida-Infektionen mit 22,8\% im Anidulafungin-Arm eine Gesamtmortalitätsrate deutlich unter $30 \%$ beobachtet worden.

Anidulafungin war insbesondere bei der häufigsten pathogenen Candida-Spezies C. albicans - der «Domäne von Fluconazol» - substanziell besser wirksam als die Vergleichssubstanz. Die klinische
Erfolgsrate betrug 81 vs. $62 \%$ ( $\mathrm{p}=0,02)$, die Eradikationsrate 95 vs. $81 \%$; $(\mathrm{p}=0,01)$. Auch bei den meisten anderen Erregern erzielte Anidulafungin höhere Eradikationsraten als Fluconazol. Bei C. parapsilosis wurde kein signifikanter Unterschied in der Wirksamkeit zu Fluconazol verzeichnet. Nach Aussage von Rotstein erwies sich Anidulafungin als gut verträglich und war mit signifikant weniger Leberwerterhöhungen assoziiert im Vergleich zu Fluconazol. Insgesamt verfüge die neue Substanz wegen der fehlenden hepatischen Verstoffwechselung über ein besonders günstiges Interaktionsprofil. Klinisch relevante Wechselwirkungen sind für Anidulafungin nicht beschrieben. Rotstein resümierte, Echinocandine seien angesichts der aktuellen Datenlage die zu präferierende Initialtherapie bei Candidämie, was sich in den aktuellen IDSA(Infectious Diseases Society of America)Leitlinien widerspiegelt - wobei für Anidulafungin die Überlegenheit gegenüber Fluconazol belegt sei.

\section{Frühe Initialtherapie verbessert die Prognose} der Patienten

Eine frühe, wirksame Initialtherapie ist entscheidend für die Prognose des Krankheitsverlaufes und senkt die Mortalität der Patienten, wie Prof. Olivier Lortholary, Institut Pasteur, Paris, in seinem Vortrag erläuterte [3]. Daher gebe es bei schwer kranken Patienten auf Intensivstationen keinen Spielraum für Irrtümer, die Initialtherapie müsse verlässlich greifen, betonte Prof. Bart-Jan Kullberg, Universität Nijmegen/ Niederlande, auf dem Meet-the-Experts Workshop «Treatment of Candidemia» [4].Die Wirksamkeit einer Therapie mit höher dosiertem Fluconazol $(800 \mathrm{mg} / \mathrm{d})$ sei nicht belegt, die klinische Erfahrung lasse auf eine schlechtere Wirksamkeit schließen als beim Einsatz von Echinocandinen, wobei hierzu jedoch keine Vergleichsstudien vorliegen.
Quellen

1 Reboli AC, Rotstein C, Pappas PG, Chapman SW, Kett DH, Kumar D, Betts R, Wible M, Goldstein BP, Schranz J, Krause DS, Walsh TJ; Anidulafungin Study Group. Anidulafungin versus fluconazole for invasive candidiasis. N Engl J Med. 2007; 356:2472-2482.

2 Rotstein C. Anidulafungin - A New Option for Candidemia. Presented at Integrated symposium 5 (sponsored by Pfizer): Managing Candidemia What's New in 2007? Trends in Medical Mycology 2007; Turin, 30. October 2007

3 Lortholary O. Treating Candidemia in 2007 Therapeutic Strategies and Challenges. Presented at Integrated symposium 5 (sponsored by Pfizer): Managing Candidemia - What's New in 2007? Trends in Medical Mycology 2007; Turin, 30. October 2007.

4 Kullberg BJ. Treatment of candidemia, including catheter management. Presented at Meet the expert session M11. Trends in Medical Mycology 2007; Turin, 31. October 2007.

Weitere Informationen bei Pfizer Deutschland GmbH Franziska Theobald Tel. +49 721 6101-9466 Fax +49 721 6010-9466 franziska.theobald@pfizer.com

\section{PharmaTicker+++ PharmaTicker+++ PharmaTicker+++ PharmaTicker+++}

Pfrimmer Nutricia GmbH. Unter der Internetadresse www.krebsundernaehrung.de steht Krebspatienten und deren Angehörigen, aber auch Ärzten ab sofort ein neuer Onlineservice zur Verfügung. Interessierte finden hier fachliche Informationen und Erklärungen zum Thema Ernährungstherapie im Rahmen der Krebsbehandlung.

Weitere Informationen bei

Sudler \& Hennessey GmbH

Jessica Grünschläger

Tel. +49 6102 7993-126, -301

Jessica.Gruenschlaeger@sudler.com
Merck Pharma GmbH. Neue Studiendaten von Adam R. et al. zeigen, dass eine Therapie mit Erbitux mehr Resektionen bei Patienten mit metastasiertem kolorektalen Karzinom ermöglicht, die auf eine Chemotherapie nicht mehr angesprochen hatten. Die Ergebnisse wurden im Oktober 2007 im Journal of Clinical Oncology veröffentlicht.

Weitere Informationen bei IntraMedic GmbH Yvonne Söltl

Tel. +49 6102 7993-345

Yvonne_Soelt!@yr.com
Pharmion Corporation. Das Unternehmen gab im Dezember 2007 die vollständigen Ergebnisse einer multizentrischen, internationalen, randomisierten Phase-III-Studie bekannt, in der Azacitidin (Vidaza ${ }^{\circledR}$ ) bei der Behandlung von Patienten mit Hochrisiko-MDS (Myelodysplastischem Syndrom) untersucht wurde. Die Daten belegen, dass Azacitidin im Vergleich zu konventionellen Therapien einen signifikanten Überlebensvorteil erzielt.

Ogilvy Healthworld GmbH

Marina Mertens

Tel. +49 211 49700-519, Fax +49 211132807 marina.mertens@ogilvy.com 


\section{Velcade $^{\circledR}$ (Bortezomib) auch in der Therapie niereninsuffizienter Mye- lompatienten effektiv und verträglich}

Neueste beim Internationalen Myelomworkshop auf Kos/Griechenland (25.-30.06.2007) vorgestellte Daten zeigen: Bortezomib (Velcade $^{\circledR}$ ), die stärkste Monosubstanz beim multiplen Myelom, ist unter entsprechender medizinischer Überwachung auch bei Niereninsuffizienz eine verträgliche und effektive Therapieoption [1]. So konnten in einer Subgruppenauswertung der Phase-III-Studie APEX auch bei einer eingeschränkten Nierenfunktion die Zeit bis zur erneuten Progression sowie das Gesamtüberleben im Vergleich zu dem bisherigen Standard Dexamethason signifikant verlängert werden [2]. Damit bestätigt die Analyse die bisherigen Ergebnisse kleinerer Studien zum Nutzen des Proteasom-Inhibitors in diesem Patientenkollektiv. Im Oktober 2007 erteilte bereits die US-amerikanische Zulassungsbehörde (FDA) eine Indikationserweiterung für den Einsatz von Bortezomib in der Standarddosierung bei niereninsuffizienten Patienten inklusive solcher mit Dialysepflicht.

Viele Myelompatienten haben aufgrund ihrer Erkrankung eine eingeschränkte Nierenfunktion und damit einhergehend eine negative Prognose in Bezug auf das Überleben. Um so wichtiger ist es deshalb zu erforschen, inwiefern Medikamente zur Behandlung des multiplen Myeloms auch in dieser Gruppe von $\mathrm{Pa}-$ tienten gut anwendbar sind.

Dass Bortezomib auch bei Niereninsuffizienz gut verträglich ist, zeigten zunächst Subgruppenanalysen der SUMMIT- und CREST-Studien [3] mit zum Teil reduzierter Anfangsdosis $\left(1,0 \mathrm{mg} / \mathrm{m}^{2} \mathrm{KOF}\right)$ sowie eine Dosiseskalationsstudie von Mulkerin [4] Letztere ermittelte bei 51 Patienten mit unterschiedlicher
Einschränkung der Nierenfunktion die Standarddosierung von $1,3 \mathrm{mg} / \mathrm{m}^{2}$ bei einer Kreatinin-Clearance von größer gleich $20 \mathrm{ml} /$ $\mathrm{min} / 1,73 \mathrm{~m}^{2}$ als gut tolerierbar. Insgesamt musste hier bei keinem Patienten die Therapie aufgrund der Niereninsuffizienz abgebrochen werden [4].

Zudem konnte bereits eine kleinere Untersuchung den Nutzen bei stark niereninsuffizienten Erkrankten mit einhergehender Dialysepflicht (23 der 24 eingeschlossenen Studienteilnehmer waren hiervon betroffen) aufzeigen: Von 20 auswertbaren Patienten sprachen $15(75 \%)$ auf die Bortezomib-Behandlung an, davon $30 \%$ mit einer kompletten oder nahezu kompletten Remission [5]. Als positiver Nebeneffekt der Therapie wurden drei Patienten wieder unabhängig von der Dialyse.

\section{Wirksamkeit bei eingeschränkter Nierenfunktion}

Dass Bortezomib bei Patienten mit Niereninsuffizienz nicht nur ebenso gut verträglich ist wie bei intakter Nierenfunktion, sondern auch langfristig von Vorteil ist, legt die aktuelle Subgruppenauswertung der APEX-Studie von San Miguel et al. [2] nahe: Hier wurden die Ergebnisse der ein- bis dreifach vortherapierten, randomisiert mit Bortezomib ( $\mathrm{n}=$ $333)$ oder Dexamethason $(n=336)$ behandelten Patienten anhand ihrer Kreatinin-Clearance analysiert. Wie die Resultate zeigten, war der Proteasom-Inhibitor im direkten Vergleich mit Dexamethason sowohl beim progressionsfreien Überleben bei Patienten mit moderater bis schwerer Niereninsuffizienz $(\mathrm{CrCl} \leq 50 \mathrm{~mL} / \mathrm{min})$ mit 4,9 vs. 2,8 Monaten $(\mathrm{p}=0,02)$ als auch bei geringer bis keiner Insuffizienz ( $>50 \mathrm{~mL} / \mathrm{min}$ ) mit 6,2 vs. $3,5 \mathrm{Mo}-$ naten $(\mathrm{p}<0,0001)$ signifikant überlegen. Ebenfalls bessere Ergebnisse erzielte Borte- zomib in beiden Subgruppen auch im Hinblick auf das Gesamtüberleben: Bei einer Kreatinin-Clearence kleiner gleich $50 \mathrm{~mL} / \mathrm{min}$ mit 22,8 vs. 12,3 Monaten $(\mathrm{p}=0,09)$ und bei $>50 \mathrm{~mL} / \mathrm{min}$ mit 30,0 vs. 25,3 Monaten $(\mathrm{p}=$ $0,09)$ unter Dexamethason. Als einziger Wirkstoff mit nachgewiesenem Überlebensvorteil in diesem Patientenkollektiv konnte Bortezomib damit teilweise die schlechte Prognose kompensieren, die mit einer Nierenfunktionsstörung beim rezidivierten multiplen Myelom einhergeht. Der Wirkstoff ist dementsprechend unter medizinischer Überwachung eine wichtige Behandlungsoption für diese Patienten.

\section{Referenzen}

1 Richardson P, et al.: Abstract \# 0224 in Haematologica 2006, 91.(Suppl 1), Poster, EHA 2006, Amsterdam, 15.-18.06.2006.

2 San Miguel J-F, et al.: Abstract \# PO-1114, International Myeloma Workshop 2007, Kos, 25.-30. Juni 2007.

3 Jagannath S, et al.: Cancer 2005;103:

1195-1200.

4 Mulkerin D, et al.: J Clin Oncol 2006;25: $87 \mathrm{~s}$.

5 Chanan-Khan A, et al.: Blood 2007;109: 2604-2606.

Weitere Informationen bei SanCom $\mathrm{GmbH}$

Yasmin König

Tel. +49 6123 70558-38, Fax -69

koenig@sancom-ccs.de

\section{PharmaTicker+++ PharmaTicker+++ PharmaTicker+++ PharmaTicker+++}

GlaxoSmithKline GmbH. Nachdem erste Resultate aus klinischen Studien mit dem Impfstoff MAGE-A3-ASCI bei nicht-kleinzelligem Lungenkarzinom vorliegen, hat das Unternehmen eine weltweite, multizentrische, placebokontrollierte, randomisierte Studie der Phase III gestartet. Für Deutschland werden noch Studienteilnehmer gesucht.

Weitere Informationen bei

GlaxoSmithKline GmbH \& Co. KG

Arnd Prilipp

Tel. +49 89 36044-8117, Fax -8066

arnd.prilipp@gsk.com
Wyeth Pharma GmbH. Im November 2007 erhielt der mTOR-Inhibitor Temsirolimus (Handelsname Torise ${ }^{\circledR}$ ) von der europäischen Kommission die Zulassung zur First-line-Therapie des fortgeschrittenen Nierenzellkarzinoms bei Patienten mit hohem Risiko. Basis der Entscheidung ist ein signifikanter Überlebensvorteil gegenüber dem bisherigen Standard Interferon alfa, der in der Studie ARCC (Global Advanced Renal Cell Carcinoma Trial) gezeigt wurde.

SanCom GmbH

Yasmin König

Tel. +49 6123 70558-35, Fax -69

koenig@ sancom-ccs.de essex pharma GmbH. Das orale Alkylans Temozolomid (TEMODAL ${ }^{\circledR}$ ) steht seit Oktober 2007 in zwei zusätzlichen Wirkstärken von $140 \mathrm{mg}$ und 180 $\mathrm{mg}$ Hartkapseln zur Verfügung. Damit ist das Präparat nun in insgesamt sechs Dosierungsstärken erhältlich. Durch die flexiblere Umsetzung der Dosierung und mehr Einnahmekomfort für den Patienten wird die Anwendung deutlich erleichtert.

Weitere Informationen bei

Medizin \& Markt GmbH

Franziska Wagenschwanz

Tel. +49 89 3-8393022, -39075

wagenschwanz@medizin-und-markt.de 
Tasigna $^{\circledR}$ (Nilotinib) erhält in der Europäischen Union die Zulassung für die Zweitlinientherapie von CML-

\section{Patienten}

Tasigna ${ }^{\circledR}$ (Nilotinib) hat Ende November 2007 in der Europäischen Union die Zulassung als neue Therapie für erwachsene Patienten mit Philadelphia-Chromosom positiver $(\mathrm{Ph}+)$ chronischer myeloischer Leukämie (CML) in chronischer und akzelerierter Phase erhalten, die eine Resistenz oder Unverträglichkeit gegenüber einer früheren Behandlung einschließlich Glivec $^{\circledR}$ (Imatinib) aufweisen.

Tasigna hemmt gezielt und mit hoher Affinität das BCR-ABL-Protein, das durch das abnormale Philadelphia-Chromosom in Krebszellen produziert wird. BCR-ABL gilt als Hauptursache für die Überproduktion von Krebs verursachenden Leukozyten bei Patienten mit Ph+ CML.

Tasigna wurde so konzipiert, dass es BCR-ABL noch selektiver hemmt als Glivec. Das neue Medikament reduziert die Anzahl der Zellen, die das abnorme Philadelphia-Chromosom tragen, bei $57 \%$ der Patienten in der chronischen Phase der Erkrankung. Die meisten Patienten erreichen dieses Ansprechen innerhalb von 3 Monaten nach Beginn der Therapie mit Tasigna und sprechen nachhaltig darauf an (mediane Dauer noch nicht erreicht) [1].

\section{Daten der Zulassungsstudie}

Die Zulassung von Tasigna in der Europäischen Union basiert auf einer Zulassungsstudie, in der die zytogenetischen Ansprechraten des Medikaments (Reduktion oder Elimination des Philadelphia-Chromosoms) und die

\section{Hintergrund chronische myeloische Leukämie}

Die chronische myeloische Leukämie ist eine der vier häufigsten Leukämieformen, auf sie entfallen ca. $15 \%$ aller Leukämiefälle weltweit [3]. In Deutschland erkranken etwa 12.000 Menschen jährlich an Leukämie. Bei rund 1.500 Patienten lautet die Diagnose «chronische myeloische Leukämie» (CML). Etwa 95\% der CML-Patienten tragen das Philadelphia-Chromosom. Unbehandelt schreitet die chronische myeloische Leukämie innerhalb von 3-5 Jahren von einer anfänglichen chronischen Phase über eine Übergansphase (beschleunigte oder akzelerierte Phase) bis hin zu einer schnellen, oftmals tödlichen Form, der Blastenkrise. Glivec gilt als Goldstandard in der Primärtherapie der $C M L$.
Abb. 1. Wirkmechanismus von Nilotinib (Tasigna).

Abb. 2. Die Rolle von BCR-ABL bei der malignen Transformation und Krankheitsprogression bei CML.
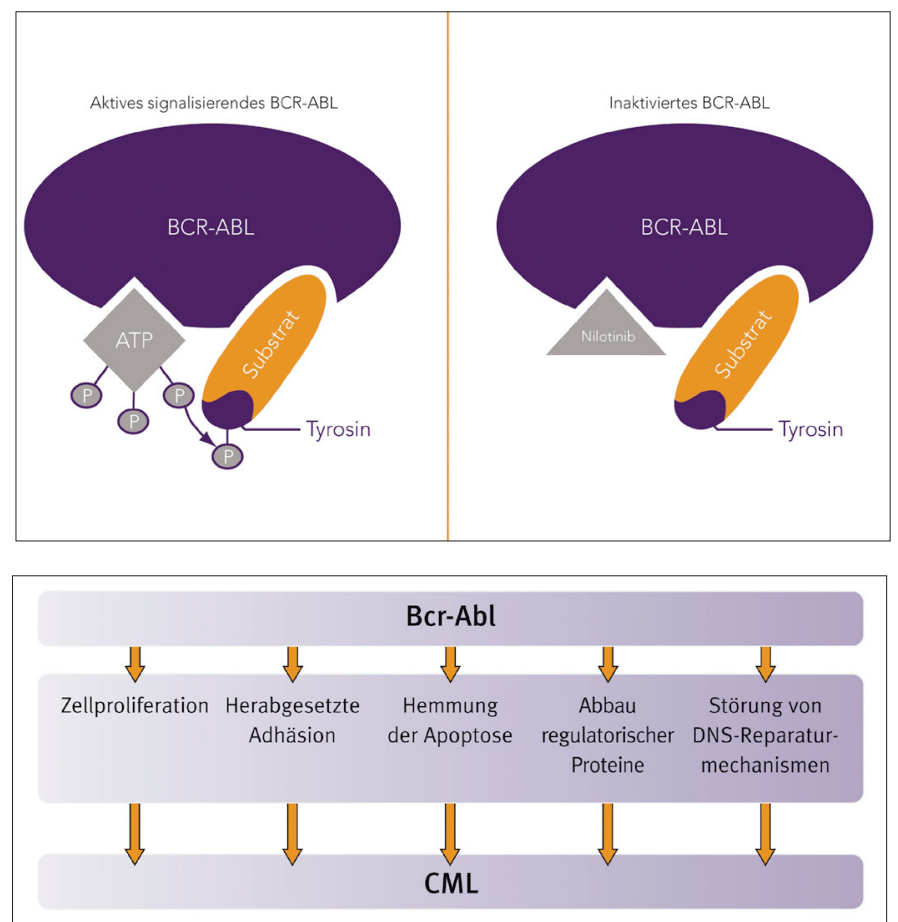

bestätigten hämatologischen Ansprechraten (Normalisierung der Leukozytenzahl) bei $\mathrm{Ph}+\mathrm{CML}-\mathrm{Patienten}$ in chronischer und akzelerierter Phase mit Glivec-Resistenz oder -Unverträglichkeit beurteilt wurden.

Ein gutes zytogenetisches Ansprechen unter Tasigna zeigten 57\% der Patienten in chronischer Phase. 77\% der Patienten in der chronischen Phase, die zu Beginn der Studie kein komplettes hämatologisches Ansprechen aufwiesen, erreichten ein komplettes hämatologisches Ansprechen in einer medianen Zeit von 1 Monat [1].

Ein gutes zytogenetisches Ansprechen unter Tasigna zeigten $31 \%$ der Patienten in akzelerierter Phase. Ein bestätigtes hämatologisches Ansprechen wurde bei $54 \%$ der Patienten in der akzelerierten Phase beobachtet. Die meisten dieser Patienten erreichten die hämatologische Remission unter Tasigna rasch (Median 1,0 Monate) und nachhaltig (Median noch nicht erreicht) [2]. Tasigna zeigte in der chronischen und akzelerierten Phase eine gute Verträglichkeit [1, 2].

\section{Zulassungsstatus von Tasigna}

Tasigna ist in 37 Ländern einschließlich aller Länder der Europäischen Union sowie Island und Norwegen, den Vereinigten Staaten und der Schweiz zugelassen. Der Antrag für die Zulassung in Japan wurde im Juni 2007 eingereicht

In den Ländern, in denen eine Zulassung vorliegt, ist Tasigna für die Behandlung von Erwachsenen mit Ph+ CML in der chronischen und akzelerierten Phase mit Resistenz oder
Unverträglichkeit gegenüber einer Vorbehandlung einschließlich Glivec indiziert. Die Wirksamkeit von Tasigna basiert auf hämatologischen und zytogenetischen Ansprechraten. Die Zulassung von Tasigna durch die US Food and Drug Administration (FDA) im Oktober 2007 basiert auf derselben klinischen Zulassungsstudie, die der EU-Zulassung zu Grunde liegt, allerdings wurde eine unterschiedliche Analyse durchgeführt. Dadurch können die Ansprechraten leicht voneinander abweichen.

Seit Anfang 2008 ist Tasigna in Deutschland verfügbar.

\section{Referenzen}

1 Kantarjian H et al., ASH 2007, Abstr. 735 und Oral Presentation

2 Le Coutre P et al., ASH 2007, Abstr. 471 und Oral Presentation

3 Faderl S, et al.: The biology of chronic myeloid leukemia. N Engl J Med 1999;341:164172.

Weitere Informationen bei

Dr. Irene Roth

Novartis Pharma GmbH

Leiterin Kommunikation Onkologie

Tel +49 91127312643

Fax +4991127311102

irene.roth@novartis.com

\section{KARGER () 2008 S. Karger GmbH, Freiburg}

